\title{
Motivic Donaldson-Thomas invariants for the one-loop quiver with potential
}

\author{
BEN DAVISON \\ SVEN MEINHARDT
}

\begin{abstract}
We compute the motivic Donaldson-Thomas invariants of the one-loop quiver, with an arbitrary potential. This is the first computation of motivic Donaldson-Thomas invariants to use in an essential way the full machinery of $\widehat{\mu}$-equivariant motives, for which we prove a dimensional reduction result similar to that of Behrend, Bryan and Szendrói in their study of degree-zero motivic Donaldson-Thomas invariants. Our result differs from theirs in that it involves nontrivial monodromy.
\end{abstract}

$14 \mathrm{~N} 35 ; 14 \mathrm{D} 23$

\section{Introduction}

Donaldson-Thomas invariants were first introduced by R Thomas [24] to count stable bundles and sheaves on a Calabi-Yau 3-fold. A few years later, D Joyce [9; 10; 11; $12 ; 13]$ and Y Song [14] generalized the definition to much more general situations using results of K Behrend [1]. Shortly after this, M Kontsevich and Y Soibelman [15; 16] came up with an alternative definition which turns out to be equivalent to the one given by Joyce. It has been realized subsequently by several people that the DonaldsonThomas invariants should be of motivic origin; in other words, they should be Euler characteristics of certain motives. Among several papers giving a definition of motivic Donaldson-Thomas invariants (at least in special cases), we will basically follow Joyce, but the reader is also encouraged to consult Behrend, Bryan and Szendröi [2], Kontsevich and Soibelman [17] and Mozgovoy [22]. There are only a few nontrivial examples for which motivic Donaldson-Thomas invariants have been computed for all classes in the Grothendieck group of the Calabi-Yau 3-category in question, though let us mention the papers Behrend, Bryan and Szendröi [2] and Morrison, Mozgovoy, Nagao and Szendröi [21]. We will start from the rather trivial example of coherent sheaves of dimension zero on $\mathbb{A}_{k}^{1}$ with $k=\bar{k}$ and char $k=0$ or, equivalently, finite-dimensional representations of $k[t]$, ie finite-dimensional representations of the one-loop quiver. The corresponding category is of homological dimension one but is also the heart of a 
bounded $t$-structure in a 3-dimensional Calabi-Yau category. The motivic DonaldsonThomas invariants for this example are well-known and given by

$$
\Omega_{n}= \begin{cases}\mathbb{L}^{1 / 2} & \text { for } n=1 \\ 0 & \text { otherwise }\end{cases}
$$

where $\mathbb{L}^{1 / 2}=1-\left[\mu_{2}\right]$ denotes a square root of the motive $\mathbb{L}$ of $\mathbb{A}_{k}^{1}$ in $\mathrm{K}^{\hat{\mu}}(\operatorname{Var} / k)$ having Euler characteristic -1 . Here $\left[\mu_{d}\right]$ denotes the $\widehat{\mu}$-equivariant motive of the set of $d^{\text {th }}$ roots of unity with obvious $\widehat{\mu}$-action. We will modify this example by considering a nonzero "potential" $W \in k[t]$ and by requiring that the sheaves are annihilated by multiplication with $W^{\prime}=d W / d t$, ie are supported on $\operatorname{Spec}\left(k[t] /\left(W^{\prime}\right)\right)$. In the language of representations, the operator $A$ given by $t$ has to satisfy $W^{\prime}(A)=0$. This class of examples provides the first case in which the full machinery of $\widehat{\mu}-$ equivariant motives has to be applied. Here is our main result.

Theorem 1.1 For $W \in k[T]$, let $W^{\prime}=c \prod_{i=1}^{r}\left(t-a_{i}\right)^{d_{i}-1}$ be the prime decomposition of $W^{\prime}$ into linear factors with $c \in k^{\times}, 1<d_{i} \in \mathbb{N}$ and $a_{i} \in k$ for all $1 \leq i \leq r$. Define the Donaldson-Thomas invariants $\Omega_{\vec{n}} \in \mathrm{K}^{\hat{\mu}}\left(\mathrm{St}^{\text {aff }} / k\right)$ for any $r$-tuple $\left(n_{1}, \ldots, n_{r}\right) \in \mathbb{N}^{r}$ as in Sections 2 and 4. Then

$$
\Omega_{\vec{n}}= \begin{cases}\mathbb{L}^{-1 / 2}\left(1-\left[\mu_{d_{i}}\right]\right) & \text { for } \vec{n}=\vec{e}_{i} \text { being the } i^{\text {th }} \text { basis vector }(1 \leq i \leq r) \\ 0 & \text { otherwise. }\end{cases}
$$

In particular, $\Omega_{\vec{n}}$ is in the image of $\mathrm{K}^{\widehat{\mu}}(\operatorname{Var} / k)\left[\mathbb{L}^{-1 / 2}\right]$ in $\mathrm{K}^{\widehat{\mu}}\left(\mathrm{St}^{\text {aff }} / k\right)$.

By taking the Euler characteristic of the nontrivial $\Omega_{\vec{n}}$, we end up with the Milnor numbers $d_{i}-1$ of $W$ at the critical points $a_{i} \in \mathbb{A}_{k}^{1}$.

Using a standard wall-crossing argument for computing Donaldson-Thomas invariants (see Lemma 4.1) and the isomorphism $\operatorname{Hilb}^{n} \mathbb{A}_{k}^{1} \cong \operatorname{Sym}^{n} \mathbb{A}_{k}^{1}$, the main theorem would actually be an easy application of the following claim concerning the commutativity of the motivic vanishing cycle and the functor $\mathrm{Sym}^{n}$.

Claim 1.2 Let $f: X \longrightarrow \mathbb{A}_{k}^{1}$ be a regular map on a smooth variety of dimension $d$. Denote by $X_{0}$ the fiber over zero. For any $n \geq 0$ there is an obvious map

$$
\operatorname{Sym}_{+}^{n}(f): \operatorname{Sym}^{n} X \stackrel{\operatorname{Sym}^{n}(f)}{\longrightarrow} \operatorname{Sym}^{n} \mathbb{A}_{k}^{1} \stackrel{+}{\rightarrow} \mathbb{A}^{1} \text {. }
$$

If we consider the motivic vanishing cycles

$$
\phi_{f} \in \mathrm{K}^{\widehat{\mu}}\left(\operatorname{Var} / X_{0}\right)\left[\mathbb{L}^{-1 / 2}\right] \quad \text { and }\left.\quad \phi_{\operatorname{Sym}_{+}^{n}(f)}\right|_{\operatorname{Sym}^{n} X_{0}} \in \mathrm{K}^{\widehat{\mu}}\left(\operatorname{Var} / \operatorname{Sym}^{n} X_{0}\right)\left[\mathbb{L}^{-1 / 2}\right]
$$


as elements of the $\lambda$-ring $\mathrm{K}^{\widehat{\mu}}\left(\operatorname{Var} / \operatorname{Sym} X_{0}\right)\left[\mathbb{L}^{-1 / 2}\right]$ via the obvious inclusions, we get for any $n \geq 0$ the equation

$$
\left.\mathbb{L}^{n d / 2} \phi_{\operatorname{Sym}_{+}^{n}(f)}\right|_{\operatorname{Sym}^{n} X_{0}}=\sigma^{n}\left(\mathbb{L}^{d / 2} \phi_{f}\right),
$$

with $\sigma^{n}(-)(n \in \mathbb{N})$ defined by the $\lambda$-ring structure introduced in the text.

The attentive reader might have realized that $\phi_{\operatorname{Sym}_{+}^{n}(f)}$ is only defined for smooth varieties $\operatorname{Sym}^{n} X$, while $\operatorname{Sym}^{n} X$ is in general not smooth. There is, however, a similar form with $\operatorname{Sym}^{n} X$ replaced by the smooth Deligne-Mumford stack $\mathcal{S y m}^{n} X:=$ $X^{n} / S_{n}$. We prove this "stacky" version, which can also be seen as a generalization of the famous Thom-Sebastaini theorem, in a forthcoming paper. For the purposes of the present paper we take $X=\mathbb{A}_{k}^{1}$, so the smoothness issue doesn't arise.

Our strategy to prove the main result is to replace the vanishing cycle with the (integral over) the much simpler vanishing cycle $\phi_{f}^{\mathrm{eq}}$ defined only for $\mathbb{G}_{m}$-equivariant functions. We then again use the wall-crossing argument of Lemma 4.1 to reduce our main theorem to Claim 1.2, but for the equivariant vanishing cycle. This equivariant vanishing cycle is much easier to work with; in particular, the above claim follows straight from the definition of the $\lambda$-ring structure.

In order to pass to the equivariant vanishing cycle from the ordinary one we will make use of the following theorem, which says that both versions of the vanishing cycle coincide (after integration) for certain $\mathbb{G}_{m}$-equivariant functions. The proof has been sketched to the second author by Dominic Joyce in a private communication. All credits are, therefore, attributed to him and all errors to us.

Theorem 1.3 Let $X$ be a smooth variety with $\mathbb{G}_{m}$-action such that every point has an open neighborhood isomorphic to $\mathbb{A}_{k}^{r} \times Z$ for some smooth $Z$ with $\mathbb{G}_{m}$ acting via $g \cdot\left(v_{1}, \ldots, v_{r}, z\right)=\left(g v_{1}, \ldots, g v_{r}, z\right)$ for all $g \in \mathbb{G}_{m},\left(v_{1}, \ldots, v_{r}\right) \in \mathbb{A}_{k}^{r}$ and $z \in Z$. Let $f: X \rightarrow \mathbb{A}_{k}^{1}$ be a $\mathbb{G}_{m}$-equivariant morphism of degree $d>0$, ie $f(g \cdot x)=g^{d} f(x)$ for all $g \in \mathbb{G}_{m}, x \in X$, and let $\widehat{\mu}$ act on $f^{-1}(1)$ via $\mu_{d}$ and trivially on $f^{-1}(0)$. Then

$$
\int_{X} \phi_{f}=\mathbb{L}^{-\operatorname{dim} X / 2}\left(\left[f^{-1}(0)\right]-\left[f^{-1}(1)\right]\right) \quad \text { in } \mathrm{K}^{\hat{\mu}}(\operatorname{Var} / k)\left[\mathbb{L}^{-1 / 2}\right] \text {. }
$$

This result should be compared to a similar result in Behrend, Bryan and Szendröi [2], where the assumptions on the action are less strict but the assumptions on $f$ are much more restrictive. Returning to Claim 1.2, and considering the situation of particular interest for this paper, in which $X=\mathbb{A}_{k}^{1}, f=x^{d}$, we see that the claim would be a special case of the above theorem, but with weights $w_{i}=i$ for $i=1, \ldots, n$, instead of all weights $w_{i}$ equal to 1 . 
Having this theorem at hand, one only has to spell out the definitions and to prove a kind of perturbation lemma allowing us to reduce ourselves to homogeneous potentials in order to give a proof of Theorem 1.1.

Acknowledgments The first author would like to thank Balázs Szendrôi for teaching him about motivic vanishing cycles, and for his support, in general, over the last few years. The first author's work was conducted partly during stays at the University of British Columbia, where he would like to thank everyone, especially Jim Bryan, for providing wonderful research conditions, and secondly at the University of Strathmore, Nairobi, which he would also like to thank for providing an excellent environment for mathematics. Finally, he would like to thank Ezra Getzler, for stimulating conversations about lambda rings and motives. As mentioned earlier, the second author is more than grateful to Dominic Joyce, not only for sharing his ideas leading to the proof of Theorem 1.3, but also for his ceaseless patience in teaching and explaining the deep theory of motivic Donaldson-Thomas invariants to him. Without this, the following paper would not exist at all. The second author would also like to thank Maxim Kontsevich for inviting him to the IHES and for some helpful discussions clarifying the big picture. The second author's research was supported by the EPSRC grants EP/D07790/1 and EP/G068798/1, by the IHES, notably by the Klaus Tschira foundation, and finally by the University of Bonn and the SFB/TR 45 .

\section{Background and conventions}

\subsection{Naive motives}

Let $\mathfrak{B}$ be an Artin stack, locally of finite type over $k$, with an action of an algebraic group $G$. We always assume that all $G$-actions on all schemes are good, in the sense that every point has an open affine $G$-equivariant neighborhood.

Definition 2.1 Define $\mathrm{K}_{0}^{G}(\operatorname{Var} / \mathfrak{B})$, as an abelian group, to be generated by isomorphism classes of $G$-equivariant finite-type morphisms $X \stackrel{f}{\longrightarrow} \mathfrak{B}$, where $X$ is a separated scheme, subject to the cut and paste relation

$$
[X \stackrel{f}{\rightarrow} \mathfrak{B}]=\left[U \stackrel{\left.f\right|_{U}}{\longrightarrow} \mathfrak{B}\right]+\left[Z \stackrel{\left.f\right|_{Z}}{\longrightarrow} \mathfrak{B}\right],
$$

where $Z \subset X$ is a closed $G$-equivariant subscheme with open complement $U$. If $G=\{1\}$, we write $\mathrm{K}_{0}(\operatorname{Var} / \mathfrak{B})$ for $\mathrm{K}_{0}^{G}(\operatorname{Var} / \mathfrak{B})$.

Notice that

$$
[X \stackrel{f}{\rightarrow} \mathfrak{B}]=\left[X_{\text {red }} \stackrel{f}{\rightarrow} \mathfrak{B}\right]
$$


in $\mathrm{K}_{0}(\operatorname{Var} / \mathfrak{B})$, justifying "Var" in our notation. If $(\mathfrak{B}, \mu, 0)$ is a $G$-equivariant monoid with finite-type multiplication map $\mu: \mathfrak{B} \times_{k} \mathfrak{B} \rightarrow \mathfrak{B}$, then $\mathrm{K}_{0}^{G}(\operatorname{Var} / \mathfrak{B})$ carries an associative product given by

$$
\left[X_{1} \stackrel{f_{1}}{\longrightarrow} \mathfrak{B}\right] \cdot\left[X_{2} \stackrel{f_{2}}{\longrightarrow} \mathfrak{B}\right]=\left[X_{1} \times_{k} X_{2} \stackrel{f_{1} \times f_{2}}{\longrightarrow} \mathfrak{B} \times{ }_{k} \mathfrak{B} \stackrel{\mu}{\rightarrow} \mathfrak{B}\right],
$$

with unit $[\operatorname{Spec}(k) \stackrel{0}{\rightarrow} \mathfrak{B}]$, making $\mathrm{K}_{0}^{G}(\operatorname{Var} / \mathfrak{B})$ into a ring. In particular, $\mathrm{K}_{0}(\operatorname{Var} / k)$ is a commutative ring. Below we will write $\mathbb{L}$ for the class $\left[\mathbb{A}_{k}^{1} \rightarrow \operatorname{Spec}(k)\right] \in \mathrm{K}_{0}(\operatorname{Var} / k)$. Each $\mathrm{K}_{0}^{G}(\operatorname{Var} / \mathfrak{B})$ is a module over $\mathrm{K}_{0}(\operatorname{Var} / k)$ via the action

$$
\left[X_{1} \rightarrow \operatorname{Spec}(k)\right] \cdot\left[X_{2} \stackrel{f}{\rightarrow} \mathfrak{B}\right]:=\left[X_{1} \times_{k} X_{2} \stackrel{f \circ \pi_{2}}{\longrightarrow} \mathfrak{B}\right],
$$

where $\pi_{2}$ is the projection onto $X_{2}$.

\section{Definition 2.2 Define}

$$
\mathrm{K}_{\mathrm{pre}}^{G}(\operatorname{Var} / \mathfrak{B})=\mathrm{K}_{0}^{G}(\operatorname{Var} / \mathfrak{B}) / H,
$$

where the subgroup $H$ is spanned by elements

$$
[\mathcal{X} \stackrel{\pi}{\rightarrow} \mathcal{Y} \stackrel{f}{\rightarrow} \mathfrak{B}]-\mathbb{L}^{r} \cdot[\mathcal{Y} \stackrel{f}{\rightarrow} \mathfrak{B}]
$$

for every $G$-equivariant vector bundle $\mathcal{X} \stackrel{\pi}{\rightarrow} \mathcal{Y}$ of rank $r$. We then define

$$
\begin{aligned}
\mathrm{K}^{G}(\operatorname{Var} / \mathfrak{B}) & =\lim _{\mathrm{K}_{\mathrm{pre}}}^{G}(\operatorname{Var} / \mathfrak{U}), \\
\mathrm{K}^{G}(\operatorname{Var} / \mathfrak{B})_{\mathrm{Gl}_{k}} & =\underset{\lim }{\longleftarrow}\left(\mathrm{K}_{\text {pre }}^{G}(\operatorname{Var} / \mathfrak{U})\left[\left[\mathrm{Gl}_{k}(n)\right]^{-1} \mid n \in \mathbb{N}\right]\right),
\end{aligned}
$$

where the limit is taken over all $G$-equivariant open subschemes $\mathfrak{U} \subset \mathfrak{B}$ of finite type and the obvious restriction maps (see below).

Let $\mathrm{K}_{0}^{G}\left(\mathrm{St}^{\mathrm{aff}} / \mathfrak{B}\right)$ be generated, as an abelian group, by isomorphism classes of $G-$ equivariant finite type morphisms $\mathcal{X} \stackrel{f}{\longrightarrow} \mathfrak{B}$, where $\mathcal{X}$ is an Artin stack with affine geometric stabilizers, again modulo the cut and paste relation (1). We define $\mathrm{K}^{G}\left(\mathrm{St}^{\text {aff }} / \mathfrak{B}\right)$ via the same completion as above. By a result of Kresch [18], if $\mathfrak{B}$ is of finite type, then for any $[\mathcal{X} \stackrel{f}{\longrightarrow} \mathfrak{B}] \in \mathrm{K}_{0}^{G}\left(\mathrm{St}^{\text {aff }} / \mathfrak{B}\right)$ there is a stratification $\mathcal{X}=\bigsqcup_{i} \mathcal{X}_{i}$ with each $\mathcal{X}_{i} \cong X_{i} / \mathrm{Gl}_{k}\left(n_{i}\right)$ a quotient stack, and it follows that

$$
[\mathcal{X} \stackrel{f}{\rightarrow} \mathfrak{B}]=\sum_{i}\left[X_{i} \stackrel{\left.f\right|_{\mathcal{X}_{i}}}{\longrightarrow} \mathfrak{B}\right] /\left[\mathrm{Gl}_{k}\left(n_{i}\right)\right] .
$$


Passing to the completion, it follows that for $\mathfrak{B}$, not necessarily of finite type, there is an isomorphism (see [7, Theorem 1.2])

$$
\mathrm{K}^{G}(\operatorname{Var} / \mathfrak{B})_{\mathrm{Gl}_{k}} \cong \mathrm{K}^{G}\left(\mathrm{St}^{\mathrm{aff}} / \mathfrak{B}\right) .
$$

If $f: \mathfrak{B} \rightarrow \mathcal{X}$ is a $G$-equivariant morphism, we obtain a map $f^{*}: \mathrm{K}^{G}\left(\mathrm{St}^{\text {aff }} / \mathcal{X}\right) \rightarrow$ $\mathrm{K}^{G}\left(\mathrm{St}^{\text {aff }} / \mathfrak{B}\right)$ given by $[X \rightarrow \mathcal{X}], \mapsto[X \times \mathcal{X} \mathfrak{B} \rightarrow \mathfrak{B}]$. If $f$ is of finite type, we obtain a map $\int_{f}: \mathrm{K}^{G}\left(\mathrm{St}^{\text {aff }} / \mathfrak{B}\right) \rightarrow \mathrm{K}^{G}\left(\mathrm{St}^{\text {aff }} / \mathcal{X}\right)$ by postcomposing with $f$. As a special case, for finite-type $U \subset \mathfrak{B}$ we define the map

$$
\int_{U}: \mathrm{K}^{\widehat{\mu}}\left(\mathrm{St}^{\mathrm{aff}} / \mathfrak{B}\right) \rightarrow \mathrm{K}^{\widehat{\mu}}\left(\mathrm{St}^{\mathrm{aff}} / k\right)
$$

as the composition $\int_{\tau_{U}} \circ i_{U}^{*}$, where $i_{U}: U \rightarrow \mathfrak{B}$ is the inclusion, and $\tau_{U}: U \rightarrow \operatorname{Spec}(k)$ is the structure morphism.

\section{$2.2 \lambda$-rings}

Definition 2.3 A $\lambda$-ring structure on a commutative $\operatorname{ring} R$ is given by a map

$$
\sigma: R \ni a \longmapsto \sigma(a)=\sum_{n=0}^{\infty} \sigma^{n}(a) T^{n} \in R \llbracket T \rrbracket
$$

such that these conditions hold:

- $\sigma(0)=1$.

- $\sigma(a)=1+a T \bmod T^{2}$ for all $a \in R$.

- $\sigma(a+b)=\sigma(a) \sigma(b)$ for all $a, b \in R$.

Example 2.4 Let $R \llbracket x \rrbracket\left[x^{-1}\right]$ be the ring of formal Laurent power series $f(x)=$ $\sum_{i} f_{i} x^{i}$ in one variable, such that $f_{i}$ vanishes for $-i$ sufficiently large. Assume that $R$ has a $\lambda$-ring structure. Then we equip $R \llbracket x \rrbracket\left[x^{-1}\right]$ with a continuous $\lambda$-ring structure by specifying $\sigma^{n}\left(r x^{i}\right)=\sigma^{n}(r) x^{n i}$. The reader might like to check the following claim: the subring of $R \llbracket x \rrbracket\left[x^{-1}\right]$ consisting of elements that can be written as a Taylor expansion around 0 of a rational function is closed under the operation of $\sigma^{n}$ for every $n$. This subring, therefore, also carries a $\lambda$-ring structure.

If $(\mathfrak{B}, \mu, 0)$ is a commutative $G$-equivariant monoid, then $\mathrm{K}^{G}(\operatorname{Var} / \mathfrak{B})$ carries a $\lambda$-ring structure defined by setting

$$
\sigma^{n}([X \stackrel{f}{\rightarrow} \mathfrak{B}]):=\left[X^{n} / S_{n} \stackrel{f^{n} / S_{n}}{\longrightarrow} \mathfrak{B}^{n} / S_{n} \stackrel{\mu}{\rightarrow} \mathfrak{B}\right] .
$$


Remark 2.5 When we come to define motivic vanishing cycles it will prove useful to be able to consider instead the ring $\mathrm{K}^{G}\left(\mathrm{St}^{\text {aff }} / \mathfrak{B}\right)=\mathrm{K}^{G}(\operatorname{Var} / \mathfrak{B})_{\mathrm{Gl}_{k}}$ as a $\lambda$-ring. We do this as follows. For simplicity we assume that $\mathfrak{B}$ is of finite type and encourage the reader to extend it to the general case. An element of $\mathrm{K}^{G}\left(\mathrm{St}^{\text {aff }} / \mathfrak{B}\right)$ can be written (nonuniquely) as a rational function $f(\mathbb{L})$ in $\mathbb{L}$ with $f \in \mathrm{K}^{G}(\operatorname{Var} / \mathfrak{B}) \llbracket T \rrbracket\left[T^{-1}\right]$. Then we apply the construction of Example 2.4 to obtain a new element $\sigma^{n}(f)$, again a rational function in $\mathbb{L}$. We claim that this element $\sigma^{n}(f)$ will also represent an element $\sigma^{n}(\tau) \in \mathrm{K}^{G}\left(\mathrm{St}^{\text {aff }} / \mathfrak{B}\right)$, which is independent of our choice of $f$.

Definition 2.6 A filtered $\lambda$-ring is a $\lambda$-ring $R$ with a decreasing filtration $\cdots \subset F_{1} \subset$ $F_{0}=R$ such that $F_{i} \cdot F_{j} \subset F_{i+j}$ and $\sigma^{n}\left(F_{i}\right) \subset F_{i n}$. If $R$ is complete with respect to the topology induced by $F^{\bullet}$, we define the operation Sym: $F^{1} \rightarrow R$ by setting $\operatorname{Sym}(a)=\sum_{n \geq 0} \sigma^{n}(a)$.

If $R$ is a complete filtered $\lambda$-ring, Sym: $F^{1} \rightarrow R$ is an isomorphism of groups from $\left(F^{1},+\right)$ to $\left(1+F^{1}, \cdot\right)$, the image of Sym.

Example 2.7 Given a commutative monoid $(\mathfrak{B}, \mu, 0)$ as before and a decreasing filtration $\cdots \mathfrak{B}_{1} \subset \mathfrak{B}_{0}=\mathfrak{B}$ by $G$-equivariant substacks such that $\mu\left(\mathfrak{B}_{i} \times \mathfrak{B}_{j}\right) \subset \mathfrak{B}_{i+j}$ and $\bigcap_{i} \mathfrak{B}_{i}=\varnothing$, then $\mathrm{K}^{G}\left(\mathrm{St}^{\text {aff }} / \mathfrak{B}\right)$ is a complete filtered $\lambda$-ring with filtration $F_{i}=\mathrm{K}^{G}\left(\mathrm{St}^{\text {aff }} / \mathfrak{B}_{i}\right) \hookrightarrow \mathrm{K}^{G}\left(\mathrm{St}^{\text {aff }} / \mathfrak{B}\right)$. In particular, the map Sym: $\mathrm{K}^{G}\left(\mathrm{St}^{\text {aff }} / \mathfrak{B}_{1}\right) \rightarrow$ $\mathrm{K}^{G}\left(\mathrm{St}^{\text {aff }} / \mathfrak{B}\right)$ is well-defined. A similar statement holds for $\mathrm{K}^{G}(\operatorname{Var} / \mathfrak{B})$.

Let $\hat{\mu}$ be the projective limit of the groups of roots of unity $\mu_{d} \subset k^{\times}$along the system of surjections $\mu_{d} \rightarrow \mu_{d^{\prime}}$, defined for $d^{\prime} \mid d$ by $z \mapsto z^{d / d^{\prime}}$. Let $\mathfrak{B}$ be any locally finite type $k$-scheme, which we equip with the trivial $\hat{\mu}$-action. We impose the extra condition on $\mathrm{K}_{0}^{\widehat{\mu}}(\operatorname{Var} / \mathfrak{B})$ and $\mathrm{K}_{0}^{\widehat{\mu}}\left(\mathrm{St}^{\text {aff }} / \mathfrak{B}\right)$ that all elements $[X \rightarrow \mathfrak{B}]$ satisfy the condition that $X$ has an open $\widehat{\mu}$-equivariant affine cover by varieties $U_{i}$ for which the $\hat{\mu}$-action factors through a $\mu_{d}$-action for some $d \in \mathbb{N}$. We then define $\mathrm{K}^{\widehat{\mu}}(\operatorname{Var} / \mathfrak{B})$ and $\mathrm{K}^{\widehat{\mu}}\left(\mathrm{St}^{\text {aff }} / \mathfrak{B}\right)$ as before, by passing to the completion and imposing the relation (2) for the $\hat{\mu}$-action. Note that by our condition on the $\hat{\mu}$-action, and the fact that we pass to the completion, every element of $\mathrm{K}^{\widehat{\mu}}\left(\mathrm{St}^{\text {aff }} / \mathfrak{B}\right)$ is a limit of elements of $\mathrm{K}^{\mu_{d}}\left(\mathrm{St}^{\mathrm{aff}} / \mathfrak{B}\right)$ for varying $d$.

We construct a $\lambda$-ring structure on $\mathrm{K}^{\widehat{\mu}}\left(\mathrm{St}^{\text {aff }} / \mathfrak{B}\right)$ by constructing compatible $\lambda$-ring structures on the rings $\mathrm{K}^{\mu_{d}}\left(\mathrm{St}^{\mathrm{aff}} / \mathfrak{B}\right)$.

Given an element $[X \stackrel{f}{\rightarrow} \mathfrak{B}] \in \mathrm{K}^{\mu_{d}}(\operatorname{Var} / \mathfrak{B})$ we construct the element

$$
-\left[X \times_{\mu_{d}} \mathbb{G}_{m} \stackrel{(x, z) \mapsto\left(f(x), z^{d}\right)}{\longrightarrow} \mathfrak{B} \times_{k} \mathbb{G}_{m}\right] \in \mathrm{K}^{\mathbb{G}_{m}, d}\left(\operatorname{Var} / \mathfrak{B} \times_{k} \mathbb{G}_{m}\right),
$$


where the additional superscript $d$ at $\mathrm{K}^{\mathbb{G}_{m}}$ indicates that $\mathbb{G}_{m}$ acts on $\mathfrak{B} \times_{k} \mathbb{G}_{m}$ via the trivial action on $\mathfrak{B}$ and the weight- $d$ action on $\mathbb{G}_{m}$. This defines an isomorphism which we denote

$$
\alpha: \mathrm{K}^{\mu_{d}}(\operatorname{Var} / \mathfrak{B}) \rightarrow \mathrm{K}^{\mathbb{G}_{m}, d}\left(\operatorname{Var} / \mathfrak{B} \times_{k} \mathbb{G}_{m}\right)
$$

Let $j: \mathbb{G}_{m} \rightarrow \mathbb{A}_{k}^{1}$ be the natural inclusion. Denoting by $\beta$ the composition of maps $\mathrm{K}^{\mathbb{G}_{m}, d}\left(\operatorname{Var} / \mathfrak{B} \times_{k} \mathbb{G}_{m}\right) \stackrel{\int_{\mathrm{id} \times j}}{\longrightarrow} \mathrm{K}^{\mathbb{G}_{m}, d}\left(\operatorname{Var} / \mathfrak{B} \times_{k} \mathbb{A}_{k}^{1}\right) \stackrel{q}{\rightarrow} \mathrm{K}^{\mathbb{G}_{m}, d}\left(\operatorname{Var} / \mathfrak{B} \times_{k} \mathbb{A}_{k}^{1}\right) / \mathfrak{I}_{d}$, we obtain another isomorphism. Here $\mathfrak{I}_{d}$ is spanned by elements $\left[X \times \mathbb{A}_{k}^{1} \stackrel{f \times \text { id }}{\longrightarrow}\right.$ $\left.\mathfrak{B} \times \mathbb{A}_{k}^{1}\right]$, with $\mathbb{G}_{m}$ acting on the domain via the trivial action on $X$ and the weight- $d$ action on $\mathbb{A}_{k}^{1}$, and $q$ is the quotient morphism.

Now let $\mathfrak{B}$ be a monoid. We assume as always that the map $\mu: \mathfrak{B} \times_{k} \mathfrak{B} \rightarrow \mathfrak{B}$ is finite-type. We make $\mathfrak{B} \times_{k} \mathbb{A}_{k}^{1}$ into a monoid via

$$
\left(\left(x_{1}, z_{1}\right),\left(x_{2}, z_{2}\right)\right) \mapsto\left(\mu\left(x_{1}, x_{2}\right), z_{1}+z_{2}\right) .
$$

Proposition 2.8 The ideal $\mathfrak{I}_{d}$ is a $\lambda$-ideal of the $\lambda$-ring $\mathrm{K}^{\mathbb{G}_{m}, d}\left(\operatorname{Var} / \mathfrak{B} \times_{k} \mathbb{A}_{k}^{1}\right)$, ie it is an ideal that is preserved by the operations $\sigma^{n}$. The resulting $\lambda$-ring structure on $\mathrm{K}^{\mathbb{G}_{m}, d}\left(\operatorname{Var} / \mathfrak{B} \times_{k} \mathbb{A}_{k}^{1}\right) / \mathfrak{I}_{d} \cong \mathrm{K}^{\mu_{d}}(\operatorname{Var} / \mathfrak{B})$ is compatible with the inclusions $\mathrm{K}^{\mu_{d}}(\operatorname{Var} / \mathfrak{B}) \rightarrow \mathrm{K}^{\mu_{d}^{\prime}}(\operatorname{Var} / \mathfrak{B})$ for $d \mid d^{\prime}$, and defines a $\lambda$-ring structure on $\mathrm{K}^{\widehat{\mu}}\left(\mathrm{St}^{\text {aff }} / \mathfrak{B}\right)$ by the construction of Remark 2.5 .

We can now explain the mysterious sign in (3) — it is this choice of sign that makes the above $\lambda$-ring structure an extension of the natural $\lambda$-ring structure on $\mathrm{K}(\operatorname{Var} / \mathfrak{B}) \subset$ $\mathrm{K}^{\widehat{\mu}}(\operatorname{Var} / \mathfrak{B})$, the subring of elements with trivial monodromy action.

We denote by $\mathbb{L}^{1 / 2}$ the element $1-\left[\mu_{2}\right]=\alpha^{-1} \beta^{-1} q\left[\mathbb{A}_{k}^{1} \stackrel{x \mapsto x^{2}}{\longrightarrow} \mathbb{A}_{k}^{1}\right] \in \mathrm{K}^{\mu_{2}}(\operatorname{Var} / k)$. A quick calculation shows that this is indeed a square root of $\mathbb{L}$ for the given ring structure on $\mathrm{K}^{\hat{\mu}}(\operatorname{Var} / k)$. In what follows we will abuse notation and consider elements of $\mathrm{K}^{\mathbb{G}_{m}, d}\left(\operatorname{Var} / \mathfrak{B} \times_{k} \mathbb{A}_{k}^{1}\right)$ as elements of $\mathrm{K}^{\mu_{d}}(\operatorname{Var} / \mathfrak{B})$ via the map $\alpha^{-1} \beta^{-1} q$.

\subsection{Vanishing cycles}

Below we will recall the motivic vanishing cycle $\phi_{f}$ of Denef and Loeser, associated to a function $f: X \rightarrow \mathbb{A}_{k}^{1}$ from a smooth equidimensional variety $X$. The existence of a sensible motivic construction for the motivic vanishing cycle is not a trivial matter; for instance, even in the analytic situation, for general $f$ there is no sufficiently small $\epsilon \in \mathbb{R}_{>0}$ such that, for all $\epsilon^{\prime}<\epsilon$, the element $\left[f^{-1}\left(\epsilon^{\prime}\right)\right] \in \mathrm{K}(\operatorname{Var} / \mathbb{C}$ ) (which is the naive 
guess of what the motivic vanishing cycle might be) is independent of $\epsilon^{\prime}$. But while we have all the maps to hand, let us remind the reader of the definition of the (integral over the) equivariant motivic vanishing cycle, defined in those situations for which the naive guess makes sense. For this we assume that $f$ is in fact $\mathbb{G}_{m}$-equivariant, where $\mathbb{G}_{m}$ acts on the target with weight $d$. Then

$$
\int_{X} \phi_{f}^{\mathrm{eq}}:=\mathbb{L}^{-\operatorname{dim}(X) / 2} \cdot\left[X \stackrel{f}{\rightarrow} \mathbb{A}_{k}^{1}\right] \in \mathrm{K}^{\mu_{d}}(\operatorname{Var} / k)\left[\mathbb{L}^{-1 / 2}\right] \subset \mathrm{K}^{\widehat{\mu}}(\operatorname{Var} / k)\left[\mathbb{L}^{-1 / 2}\right] .
$$

Going through the identifications made before, we can also write

$$
\int_{X} \phi^{\mathrm{eq}}=\mathbb{L}^{-\operatorname{dim}(X) / 2}\left(\left[f^{-1}(0)\right]-\left[f^{-1}(1)\right]\right),
$$

with $f^{-1}(1)$ carrying the natural induced $\mu_{d}$-action, while $\widehat{\mu}$ acts trivially on $f^{-1}(0)$. Notice that we only defined the "integral over the equivariant vanishing cycle" but not the $\phi_{f}^{\mathrm{eq}}$ themselves. There is an obvious generalization of our definition to $\mathbb{G}_{m}-$ equivariant morphisms $p \times f: X \rightarrow \mathfrak{B} \times{ }_{k} \mathbb{A}_{k}^{1}$, giving rise to

$$
\int_{p} \phi_{f}^{\mathrm{eq}}:=\mathbb{L}^{-\operatorname{dim}(X) / 2}\left[X \stackrel{p \times f}{\longrightarrow} \mathfrak{B} \times_{k} \mathbb{A}_{k}^{1}\right] \in \mathrm{K}^{\widehat{\mu}}(\operatorname{Var} / \mathfrak{B})\left[\mathbb{L}^{-1 / 2}\right] .
$$

Now let $X$ be a smooth equidimensional variety and let $f: X \rightarrow \mathbb{A}_{k}^{1}$ be a regular map. We denote by $\operatorname{Crit}(f)$ the critical locus of $f$. By replacing $X$ with a Zariski open subscheme $U \subset X$ we may assume $\operatorname{Crit}(f) \subset X_{0}:=f^{-1}(0)$. In this setup Denef and Loeser $[5 ; 6]$ constructed a motivic analogue of the sheaf of vanishing cycles associated to $f$. We briefly recall a modified version of their construction. Define $\mathcal{L}_{n}(X)$ to be the space of arcs of length $n$ in $X$, ie the scheme representing the functor $Y \mapsto \operatorname{Hom}_{\operatorname{Sch}}\left(Y \times_{k} \operatorname{Spec}\left(k[t] / t^{n+1}\right), X\right)$. There is a natural morphism $p_{n}: \mathcal{L}_{n}(X) \rightarrow X$ defined by the inclusion

$$
\operatorname{Spec}(k) \cong \operatorname{Spec}(k[t] / t) \rightarrow \operatorname{Spec}\left(k[t] / t^{n+1}\right),
$$

and we define $\left.\mathcal{L}_{n}(X)\right|_{X_{0}}$ to be the preimage of $X_{0}$ under this map. The map $f$ induces a map $\mathcal{L}_{n}(f): \mathcal{L}_{n}(X) \rightarrow \mathcal{L}_{n}\left(\mathbb{A}_{k}^{1}\right)$, and there is an isomorphism $\mathcal{L}_{n}\left(\mathbb{A}_{k}^{1}\right) \cong \mathbb{A}_{k}^{n+1}$, given by writing an arc in $\mathbb{A}_{k}^{1}$ as a polynomial $a_{0}+\cdots+a_{n} T^{n}$. Sending an arc $\gamma$ in $\mathcal{L}_{n}(X)$ to the coefficient $a_{n}$ of $\mathcal{L}_{n}(f)(\gamma)$ defines a $\mathbb{G}_{m}$-equivariant map $p_{n} \times f_{n}: \mathcal{L}_{n}(X) \longrightarrow$ $X \times_{k} \mathbb{A}_{k}^{1}$, where $\mathbb{G}_{m}$ acts on $\mathcal{L}_{n}(X)$ by rescaling $t$. We define

$$
\begin{aligned}
Z_{f}^{\mathrm{eq}}(T) & =\left.\sum_{n \geq 1}\left(\int_{p_{n}} \phi_{f_{n}}^{\mathrm{eq}}\right)\right|_{X_{0}} T^{n} \\
& =\sum_{n \geq 1} \mathbb{L}^{-(n+1) \operatorname{dim}(X) / 2}\left[\left.\mathcal{L}_{n}(X)\right|_{X_{0}} \stackrel{p_{n} \times f_{n}}{\longrightarrow} X_{0} \times_{k} \mathbb{A}_{k}^{1}\right] T^{n}
\end{aligned}
$$


in $\mathrm{K}^{\widehat{\mu}}\left(\operatorname{Var} / X_{0}\right)\left[\mathbb{L}^{-1 / 2}\right] \llbracket T \rrbracket$. Denef and Loeser prove that this is in fact a rational function in $T$ with no pole at infinity, and we define

$$
\phi_{f}:=-Z_{f}^{\mathrm{eq}}(\infty) \text {. }
$$

Our definition differs from that of Denef and Loeser by a factor $\left(-\mathbb{L}^{1 / 2}\right)^{\operatorname{dim}(X)}$. It coincides with that of Kontsevich and Soibelman [15].

We give an alternative description, also due to Denef and Loeser. Firstly, let $\pi: Y \rightarrow X$ be an embedded resolution of $X_{0}$, ie a proper morphism, which is an isomorphism away from $X_{0}$, and for which $Y_{0}:=\pi^{-1}\left(X_{0}\right)$ is a simple normal crossing divisor, ie the irreducible components of $Y_{0}$ are smooth hypersurfaces $E_{i}$ for $i$ indexed by some finite set $J$, and the $E_{i}$ are étale locally cut out by independent linear equations. Denote by $m_{i}$ the order of vanishing of $f \circ \pi$ on $E_{i}$, and for $\varnothing \neq I \subset J$ denote by $E_{I}^{\circ}$ the locally closed subscheme $\bigcap_{i \in I} E_{i} \backslash \cup_{i \notin I} E_{i}$.

The function $f \circ \pi$ defines a section of $\mathcal{O}_{Y}\left(-\sum_{i \in I} m_{i} E_{i}\right)$, and so a regular map, linear along the fibers, from the total space of $\mathcal{O}_{Y}\left(\sum_{i \in I} m_{i} E_{i}\right)$ to $\mathbb{A}_{k}^{1}$. The restriction of this bundle to $E_{I}^{\circ}$ is just $\bigotimes_{i \in I} N_{E_{i} \mid Y}^{\otimes m_{i}}$, so that the map

$$
f_{I}: N_{I}:=\left.\prod_{i \in I}\left(N_{E_{i} \mid Y} \backslash E_{i}\right)\right|_{E_{I}^{\circ}} \rightarrow \mathbb{A}_{k}^{1},
$$

defined by taking the tensor product and evaluating $f \circ \pi$, is homogeneous of degree $m_{I}=\sum_{i \in I} m_{i}$, if we let $\mathbb{G}_{m}$ act by rescaling each copy of $N_{E_{i} \mid Y}$. We denote by $\pi_{I}:\left.\mathcal{O}_{Y}\left(\sum_{i \in I} m_{i} E_{i}\right)\right|_{E_{I}^{\circ}} \rightarrow E_{I}^{\circ}$ the projection.

Theorem 2.9 In $\mathrm{K}^{\widehat{\mu}}\left(\operatorname{Var} / X_{0}\right)\left[\mathbb{L}^{-1 / 2}\right]$, we have the equality

$$
\phi_{f}=\mathbb{L}^{-\operatorname{dim}(X) / 2}\left(\left[X_{0} \stackrel{\mathrm{id}}{\rightarrow} X_{0}\right]+\sum_{\varnothing \neq I \subset J}\left[f_{I}^{-1}(1) \stackrel{\pi \circ \pi_{I} \times_{k} f_{I}}{\longrightarrow} X_{0} \times_{k} \mathbb{A}_{k}^{1}\right]\right) .
$$

In particular, the right-hand side of (5) is independent of the embedded resolution $\pi: Y \rightarrow X$.

If $X$ is smooth but not equidimensional, we extend the above expression by linearity. The following proposition follows from Theorem 2.9.

Proposition 2.10 (1) The motive $\phi_{f}$ is supported on $\operatorname{Crit}(f) \subset X_{0}$; in other words, it is contained in

$$
\mathrm{K}^{\widehat{\mu}}(\operatorname{Var} / \operatorname{Crit}(f))\left[\mathbb{L}^{-1 / 2}\right] \hookrightarrow \mathrm{K}^{\widehat{\mu}}\left(\operatorname{Var} / X_{0}\right)\left[\mathbb{L}^{-1 / 2}\right] \cdot{ }^{1}
$$

${ }^{1}$ Notice that $\mathrm{K}^{\hat{\mu}}(\operatorname{Var} / \varnothing)=\{0\}$ 
(2) If $f \equiv 0$, then $\phi_{f}=\mathbb{L}^{-\operatorname{dim} X / 2}[X \stackrel{\mathrm{id}}{\rightarrow} X]$.

(3) Let $\pi: Y \rightarrow X$ be a smooth morphism of relative dimension $r$. Then

$$
\phi_{f \circ \pi}=\mathbb{L}^{-r / 2} \pi^{*} \phi_{f} .
$$

Given a quotient stack $\mathcal{X}=X / \mathrm{Gl}_{k}(n)$ with atlas $\pi: X \rightarrow X / \mathrm{Gl}_{k}(n)$ and a regular function $f: \mathcal{X} \rightarrow \mathbb{A}_{k}^{1}$, we extend our definition of motivic vanishing cycles by putting

$$
\phi_{f}:=\left[\mathrm{Gl}_{k}(n)\right]^{-1} \mathbb{L}^{\operatorname{dim}(\pi) / 2} \int_{\pi} \phi_{f \circ \pi} \in \mathrm{K}^{\widehat{\mu}}\left(\mathrm{St}^{\mathrm{aff}} / \operatorname{Crit}(f)\right),
$$

with $\operatorname{Crit}(f)=\operatorname{Crit}(f \circ \pi) / \mathrm{Gl}_{k}(n)$, motivated by Proposition 2.10. Note that $\mathbb{L}^{-1 / 2}$ already exists in $\mathrm{K}^{\hat{\mu}}\left(\mathrm{St}^{\text {aff }} / k\right)$ since $\mathbb{L}^{1 / 2}$ and $\mathbb{L}^{-1}$ do. This definition generalizes easily to Artin stacks $\mathcal{X}$ whose connected components are quotient stacks. In particular, if $p: \mathcal{X} \rightarrow \mathfrak{B}$ is a morphism on such an Artin stack $\mathcal{X}$, then $\int_{p} \phi_{f} \in \mathrm{K}^{\widehat{\mu}}\left(\mathrm{St}^{\text {aff }} / \mathfrak{B}\right)$ is well defined.

\subsection{Quivers with potential}

Let $Q$ be a quiver, ie a directed graph with finitely many vertices and arrows. Let $W \in \mathbb{C} Q /[\mathbb{C} Q, \mathbb{C} Q]$ be a potential, ie a finite linear combination of cyclic words in the quiver, with each word only considered up to cyclic permutation. If $\gamma \in \mathbb{N} Q_{0}$ is a dimension vector, the space

$$
\operatorname{Rep}_{\gamma}(Q):=\bigoplus_{a \in Q_{1}} \operatorname{Hom}\left(\mathbb{C}^{\gamma(s(a))}, \mathbb{C}^{\gamma(t(a))}\right)
$$

is a parameter space of left $\mathbb{C} Q$-representations of dimension vector $\gamma$. Let the gauge group $G_{\gamma}:=\prod_{i \in Q_{0}} \mathrm{Gl}_{k}(\gamma(i))$ act on $\operatorname{Rep}_{\gamma}(Q)$ by change of basis on the vector space $\bigoplus_{i \in Q_{0}} \mathbb{C}^{\gamma(i)}$. Then the stack of $\gamma$-dimensional left $\mathbb{C} Q$-modules is isomorphic to the stack-theoretic quotient $\operatorname{Rep}_{\gamma}(Q) / G_{\gamma}$. We abuse notation by identifying $\mathbb{N} Q_{0}$ with the scheme $\bigsqcup_{\gamma \in \mathbb{N} Q_{0}} \operatorname{Spec}(k)$. Then the collection of morphisms

$$
\operatorname{Rep}_{\gamma}(Q) / G_{\gamma} \stackrel{\tau_{\gamma}}{\longrightarrow} \operatorname{Spec}(k)
$$

turns the stack of finite-dimensional $k Q$-modules into a stack over $\mathbb{N} Q_{0}$, and we define

$$
\operatorname{dim}: \coprod_{\gamma \in \mathbb{N} Q_{0}}\left(\operatorname{Rep}_{\gamma}(Q) / G_{\gamma} \stackrel{\tau_{\gamma}}{\rightarrow} \operatorname{Spec}(k)\right) .
$$

From the potential $W$ we obtain a function $\mathcal{W}:=\operatorname{Tr}(W)$ on this stack, and so an element

$$
\Phi_{W}:=\int_{\operatorname{dim}} \phi_{\mathcal{W}} \in \mathrm{K}^{\widehat{\mu}}\left(\mathrm{St}^{\mathrm{aff}} / \mathbb{N} Q_{0}\right) .
$$


Now $\mathbb{N} Q_{0}$ is a commutative monoid in the category of schemes, via the (finite-type) addition operation. As such, there is a $\lambda$-ring structure on $\mathrm{K}^{\widehat{\mu}}\left(\mathrm{St}^{\text {aff }} / \mathbb{N} Q_{0}\right)$, defined above. Define

$$
\left(\mathbb{N}^{Q_{0}}\right)_{m}:=\left\{\gamma \in \mathbb{N}^{Q_{0}} \mid \sum_{i \in Q_{0}} \gamma(i) \geq m\right\} .
$$

As seen in Example 2.7, the filtration $F_{m}=\mathrm{K}^{\widehat{\mu}}\left(\mathrm{St}^{\mathrm{aff}} /\left(\mathbb{N} Q_{0}\right)_{m}\right)$ turns $\mathrm{K}^{\widehat{\mu}}\left(\mathrm{St}^{\text {aff }} / \mathbb{N} Q_{0}\right)$ into a complete filtered $\lambda$-ring. The element $\Omega \in \mathrm{K}^{\widehat{\mu}}\left(\mathrm{St}^{\text {aff }} /\left(\mathbb{N} Q_{0}\right)_{\geq 1}\right)$ is defined by the expression

$$
\operatorname{Sym}\left(\frac{\Omega}{\mathbb{L}^{1 / 2}-\mathbb{L}^{-1 / 2}}\right)=\Phi_{W} .
$$

Definition 2.11 If $i_{\gamma}: \operatorname{Spec}(k) \rightarrow \mathbb{N} Q_{0}$ is the inclusion of the point $\gamma$, then we define the motivic Donaldson-Thomas invariant $\Omega_{\gamma}=i_{\gamma}^{*} \Omega \in \mathrm{K}^{\widehat{\mu}}\left(\mathrm{St}^{\mathrm{aff}} / k\right)$.

Conjecturally, the elements $\Omega_{\gamma}$ all belong to the image of $\mathrm{K}^{\widehat{\mu}}(\operatorname{Var} / k)\left[\mathbb{L}^{-1 / 2}\right] \rightarrow$ $\mathrm{K}^{\widehat{\mu}}\left(\mathrm{St}^{\text {aff }} / k\right)$. Our main theorem verifies this conjecture in the case of the one-loop quiver with arbitrary potential.

\section{Vanishing cycles for equivariant functions}

The following theorem is very useful for computing the motivic vanishing cycle in a $\mathbb{G}_{m}$-equivariant situation - it states that under special hypotheses on the $\mathbb{G}_{m}$-action of the domain of a $\mathbb{G}_{m}$-equivariant function, the motivic vanishing cycle of Denef and Loeser is the same as the naive vanishing cycle $\phi_{f}^{\mathrm{eq}}$ of (4). Let $X$ be a smooth equidimensional variety locally of finite type equipped with a $\mathbb{G}_{m}$-action. Let us assume that every closed point has an open neighborhood which as a variety with $\mathbb{G}_{m}$-action is isomorphic to $\mathbb{A}_{k}^{r} \times Z$ with $\mathbb{G}_{m}$ acting via $g \cdot\left(v_{1}, \ldots, v_{r}, z\right)=\left(g^{w_{1}} v_{1}, \ldots, g^{w_{r}} v_{r}, z\right)$ with strictly positive weights $w_{1}, \ldots, w_{r}$. In particular, $Z$ is the intersection of the neighborhood with the fixed point set $X^{\mathbb{G}_{m}}$ and, hence, smooth. Moreover, it is not difficult to see that the projection to $Z$ along $\mathbb{A}_{k}^{r}$ can be described by $\lim _{g \rightarrow 0} g \cdot x$ and extends, therefore, to a smooth map $X \rightarrow X^{\mathbb{G}_{m}}$. The assumption is not very restrictive, as any smooth projective variety with $\mathbb{G}_{m}$-action has a dense open subset satisfying our assumption by a theorem of Białynicki-Birula [3; 4]. Conjecturally, any smooth quasiprojective variety $X$ with $\mathbb{G}_{m}$-action such that $X^{\mathbb{G}_{m}}$ is connected and $\lim _{g \rightarrow 0} g \cdot x$ exists for any closed point $x$ should satisfy our assumption.

Theorem 3.1 Let $X$ be a smooth variety with $\mathbb{G}_{m}$-action satisfying the assumption mentioned above with all weights equal to 1 . Let $f: X \rightarrow \mathbb{A}_{k}^{1}$ be a $\mathbb{G}_{m}$-equivariant 
morphism of degree $d$, ie $f(g \cdot x)=g^{d} f(x)$ for all $g \in \mathbb{G}_{m}, x \in X$. Let $\hat{\mu}$ act on $f^{-1}(1)$ via $\mu_{d}$ and trivially on $f^{-1}(0)$. Then

$$
\int_{X} \phi_{f}=\int_{X} \phi_{f}^{\mathrm{eq}}=\mathbb{L}^{-\operatorname{dim} X / 2}\left(\left[f^{-1}(0)\right]-\left[f^{-1}(1)\right]\right) \quad \text { in } \mathrm{K}^{\widehat{\mu}}(\operatorname{Var} / k)\left[\mathbb{L}^{-1 / 2}\right] .
$$

Proof Let us first assume that $X=\mathbb{A}_{k}^{r} \times Z$, with $\mathbb{G}_{m}$ acting nontrivially only on the affine "fiber" $\mathbb{A}_{k}^{r}$ by scalar multiplication. Consider the blow-up $\tilde{X}$ of $Z$ in $X$, which has a natural fibration towards the exceptional divisor $\widetilde{E}_{0}$ induced by the affine fibration $X \rightarrow Z$. Moreover, the $\mathbb{G}_{m}$-action has a lift to $\tilde{X}$ with fixed point set $\widetilde{E}_{0}$ and it is not difficult to see that $\tilde{X}$ as a variety with $\mathbb{G}_{m}$-action is isomorphic to the normal bundle $N:=N_{\mathbb{E}_{0} \mid \tilde{X}}$ with $\mathbb{G}_{m}$ acting by scalar multiplication on the fibers. Denote by $\widetilde{E}_{i}$ for $i=1, \ldots, l$ the strict transforms of the irreducible components of the divisor $f^{-1}(0)$. As they are closed and $\mathbb{G}_{m}$-invariant, we get $\widetilde{E}_{i}=\left.N\right|_{\widetilde{D}_{i}}$ for the divisors $\widetilde{D}_{i}=\widetilde{E}_{0} \cap \widetilde{E}_{i}$ in $\widetilde{E}_{0}$. Note that the collection $\left(\widetilde{D}_{i}\right)_{i=1}^{l}$ might not be a normal crossing divisor in $\widetilde{E}_{0}$.

Let $\sigma: E_{0} \rightarrow \widetilde{E}_{0}$ be an embedded resolution of the $\widetilde{D}_{i}$; ie the strict transforms $D_{i}$ $(i=1, \ldots, l)$ together with the exceptional divisors $D_{l+1}, \ldots, D_{m}$ of $\sigma$ form a normal crossing divisor in $E_{0}$. Moreover, $E_{0}$ is smooth and

$$
\sigma: E_{0} \backslash \bigcup_{i=1}^{m} D_{i} \stackrel{\sim}{\longrightarrow} \tilde{E}_{0} \backslash \bigcup_{i=1}^{l} \tilde{D}_{i} .
$$

Consider the pullback $Y:=\sigma^{*} N$ and the normal crossing divisors $E_{0}, E_{i}:=\left.\left(\sigma^{*} N\right)\right|_{D_{i}}$ for $i=1, \ldots, m$ in $Y$ along with the proper morphism given by the composition $\pi: Y=\sigma^{*} N \stackrel{\sigma}{\rightarrow} N=\tilde{X} \rightarrow X$. By construction, $(f \circ \pi)^{-1}(0)=\bigcup_{i=0}^{m} E_{i}$ settheoretically, and $\pi: Y \backslash \bigcup_{i=0}^{m} E_{i} \stackrel{\sim}{\longrightarrow} X \backslash f^{-1}(0)$. We will use this embedded resolution of $X_{0}=f^{-1}(0)$ to compute $\int_{X} \phi_{f}$.

As $Y \stackrel{p}{\rightarrow} E_{0}$ is a line bundle, we get $N_{E_{0} \mid Y}=Y$ and $N_{\{0\}}=Y \backslash \bigcup_{i=0}^{m} E_{i}$. The induced map $f_{\{0\}}$ is just $f \circ \pi$, as the latter is homogeneous and $E_{0}$ is of multiplicity $d$. Moreover, after identifying $N_{\{0\}}$ with $X \backslash f^{-1}(0)$ by means of $\pi$, we get $f_{\{0\}}=f$ on $N_{\{0\}}$ and, thus, $f_{\{0\}}^{-1}(1)=f^{-1}(1)$ with $\widehat{\mu}$-action given by the natural $\mu_{d}$-action on $f^{-1}(1)$.

On the other hand, for any $i=1, \ldots, m$, we have by construction

$$
N_{E_{i} \mid Y}=p^{*} N_{D_{i} \mid E_{0}}=\left.N_{E_{i} \mid Y}\right|_{E_{0} \cap E_{i}} \times\left. N_{E_{0} \mid Y}\right|_{E_{0} \cap E_{i}}
$$

and, thus, for any $\varnothing \neq I \subset\{1, \ldots, m\}$,

$$
N_{I}=\left.\prod_{i \in I}\left(N_{E_{i} \mid Y} \backslash E_{i}\right)\right|_{E_{I}^{\circ}}=\left.\prod_{i \in I \cup\{0\}}\left(N_{E_{i} \mid Y} \backslash E_{i}\right)\right|_{E_{I \cup\{0\}}^{\circ}}=N_{I \cup\{0\}} .
$$


Moreover, by $\mathbb{G}_{m}$-equivariance of $f \circ \pi$ the induced maps $f_{I}$ and $f_{I \cup\{0\}}$ coincide, which can be checked by a local calculation. Hence, $f_{I}^{-1}(1)=f_{I \cup\{0\}}^{-1}(1)$. Unfortunately, the $\widehat{\mu}$-actions are different, but, nevertheless, the $\widehat{\mu}$-equivariant motives $\left[f_{I}^{-1}(1)\right]$ and $\left[f_{I \cup\{0\}}^{-1}(1)\right]$ coincide. To see this we choose local functions $\left(z_{i}\right)_{i \in I \cup\{0\}}$ in some $\mathbb{G}_{m}$-invariant neighborhood $V=p^{-1}\left(V \cap E_{0}\right)$ of $y \in E_{I \cup\{0\}}^{\circ}$ such that $E_{I \cup\{0\}}^{\circ} \cap V$ is the zero locus of $z_{0} \prod_{i \in I} z_{i}$. Hence,

$$
\left.\left.N_{I}\right|_{V} \cong N_{I \cup\{0\}}\right|_{V} \cong E_{I \cup\{0\}}^{\circ} \cap V \times \mathbb{G}_{m} \times \mathbb{G}_{m}^{I} \quad \text { and } \quad f_{I}=f_{I \cup\{0\}}=u z_{0}^{d} \prod_{i \in I} z_{i}^{m_{i}},
$$

with $u$ being a unit on $V, m_{i}>0$ being the multiplicities of $E_{i}$ in $(f \circ \pi)^{-1}(0)$ and with $z_{i}$ identified with the coordinates on the corresponding "normal" $\mathbb{G}_{m}$-factors. The action of $\hat{\mu}$ via $\mu_{m_{I}}$ with $m_{I}=\sum_{i \in I} m_{i}$ is given by diagonal embedding of $\mu_{m_{I}}$ into $\mathbb{G}_{m}^{I}$, and similarly for $\mu_{m_{I \cup\{0\}}}=\mu_{m_{I}+d}$.

However, we can choose an automorphism of $\mathbb{G}_{m} \times \mathbb{G}_{m}^{I}$ mapping

$$
\left(z_{i}\right)_{i \in I \cup\{0\}} \quad \text { to } \quad\left(\prod_{j \in I \cup\{0\}} z_{j}^{a_{j}^{(i)}}\right)_{i \in I \cup\{0\}},
$$

with $\left(a^{(i)}\right)_{I \cup\{0\}}$ being a basis of the group $\mathbb{Z} \times \mathbb{Z}^{I}$ of characters of $\mathbb{G}_{m} \times \mathbb{G}_{m}^{I}$ such that $a_{i}^{(0)}=m_{i} / e$ for all $i \in I \cup\{0\}$, with $e:=\operatorname{gcd}\left(m_{j} \mid j \in I \cup\{0\}\right)$ and $m_{0}=d$. After this coordinate change on $\left.N_{I}\right|_{V}$, the function $f_{I}$ is given by $u z_{0}^{e}$. Using relation (2), we see that $\left[f_{I}^{-1}(1)\right]$ is given by $(\mathbb{L}-1)^{|I|}\left[\widetilde{E}_{I \cup\{0\}}^{\circ}\right]$, with $\widetilde{E}_{I \cup\{0\}}^{\circ}$ being a Galois cover of $E_{I \cup\{0\}}^{\circ}$ with Galois group $\mu_{e}$, locally given by

$$
\left\{(z, y) \in \mathbb{A}_{k}^{1} \times E_{I \cup\{0\}}^{\circ} \cap V \mid z^{e} u(y)=1\right\} .
$$

Moreover, the group $\mu_{m_{I}}$ acts by its quotient group $\mu_{e}$. Exactly the same holds for $f_{I \cup\{0\}}^{-1}(1)$ acted on by $\mu_{m_{I \cup\{0\}}}$.

Thus, $\left[f_{I}^{-1}(1)\right]=\left[f_{I \cup\{0\}}^{-1}(1)\right]$ in $\mathrm{K}^{\widehat{\mu}}(\operatorname{Var} / k)$ and their contributions to $\int_{X} \phi_{f}$ cancel. $^{2}$ In the formula for $\int_{X} \phi_{f}$ we are left with the contribution $f_{\{0\}}^{-1}(1)=f^{-1}(1)$, which proves the theorem.

The general case is a purely combinatorial argument using the motivic behavior of the integral. Indeed, for any $\mathbb{G}_{m}$-invariant open subset $U=\mathbb{A}_{k}^{r} \times Z$ in $X$, we get by the previous arguments

$$
\int_{U} \phi_{f}=\int_{U} \phi_{\left.f\right|_{U}}=\int_{U} \phi_{\left.f\right|_{U}}^{\mathrm{eq}}=\int_{U} \phi_{f}^{\mathrm{eq}}
$$

Let us now take a general smooth variety $X$ with $\mathbb{G}_{m}$-action satisfying the assumptions of the theorem. Choose an open covering by $\mathbb{G}_{m}$-invariant subsets $U_{i}$ of the form

\footnotetext{
${ }^{2}$ Note that the projections to $X_{0}$ are different, so they do not cancel each other in the formula for $\phi_{f}$.
} 
$U_{i}=\mathbb{A}_{k}^{r_{i}} \times Z_{i}(i \in I)$. If $U_{i} \cap U_{j} \neq \varnothing$, the intersection is of the form $\mathbb{A}_{k}^{r_{i}} \times \widetilde{Z}_{i}$ for some open $\widetilde{Z}_{i} \subset Z_{i}$, as $U_{i} \cap U_{j}$ must contain the limits $\lim _{g \rightarrow 0} g \cdot x$ for all $x \in U_{i} \cap U_{j}$. By applying (7) to any nonempty intersection $U_{J}=\bigcap_{i \in J} U_{i}$, we finally get

$$
\int_{X} \phi_{f}=\sum_{U_{J} \neq \varnothing}(-1)^{|J|+1 \mid} \int_{U_{J}} \phi_{f}=\sum_{U_{J} \neq \varnothing}(-1)^{|J|+1 \mid} \int_{U_{J}} \phi_{f}^{\mathrm{eq}}=\int_{X} \phi_{f}^{\mathrm{eq}} .
$$

Unfortunately, we were not able to prove the theorem for arbitrary positive weights $w_{1}, \ldots, w_{r}>0$. Nevertheless, we conjecture that Theorem 3.1 holds also in that case.

\section{The one-loop quiver with potential}

In this section we apply the results of the previous parts to compute the motivic Donaldson-Thomas invariants of the one-loop quiver with potential $W \in k[t]$. Let

$$
\mathcal{X}=\coprod_{n \geq 0} \operatorname{Mat}_{k}(n, n) / \operatorname{Gl}_{k}(n)=\coprod_{n \geq 0} \operatorname{Rep}(Q)_{n} / G_{n}
$$

be the stack of finite-dimensional representations of the one-loop quiver $Q$, ie representations of the ring $k[t]$. Equivalently, it can be seen as the stack parametrizing zerodimensional sheaves on $\mathbb{A}_{k}^{1}$. To a given potential $W \in k[t]$ we associate, for any $n \geq 0$, the $\mathrm{Gl}_{k}(n)$-equivariant function $W_{n}$ : $\operatorname{Mat}_{k}(n, n) \ni A \longmapsto \operatorname{tr} W(A) \in \mathbb{A}_{k}^{1}$. These induce a regular function $\mathcal{W}: \mathcal{X} \rightarrow \mathbb{A}_{k}^{1}$ whose critical locus $\mathfrak{M}:=\operatorname{Crit}(\mathcal{W})$ is the stack of finite length sheaves on $\operatorname{Spec}\left(k[t] /\left(W^{\prime}\right)\right) \subset \mathbb{A}_{k}^{1}$. Consider the homomorphism $\operatorname{dim}: \mathcal{X} \rightarrow \mathbb{N}$ defined as in (6), mapping each representation to its dimension or, equivalently, each sheaf on $\mathbb{A}_{k}^{1}$ to its length. If we denote the element $[\operatorname{Spec}(k) \rightarrow 1] \in \mathrm{K}^{\widehat{\mu}}\left(\mathrm{St}^{\text {aff }} / \mathbb{N}\right)$ by $T$, we obtain the invertible power series

$$
\Phi_{W}(T):=\int_{\operatorname{dim}} \phi_{\mathcal{W}}=\sum_{n \geq 0} \frac{\int_{\mathrm{Mat}_{k}(n, n)} \phi_{W_{n}}}{\mathbb{L}^{-n^{2} / 2}\left[\mathrm{Gl}_{k}(n)\right]} T^{n}=\operatorname{Sym}\left(\frac{1}{\mathbb{L}^{1 / 2}-\mathbb{L}^{-1 / 2}} \sum_{n \geq 1} \Omega_{n} T^{n}\right)
$$

in $\mathrm{K}^{\hat{\mu}}\left(\mathrm{St}^{\mathrm{aff}} / \mathbb{N}\right)$ by Definition 2.11. The stack $\mathcal{X}$ carries a natural good $\mathbb{G}_{m}$-action given by scalar multiplication on the space of matrices. If $W$ is homogeneous we can, therefore, also consider the invertible power series

$$
\Phi_{W}^{\mathrm{eq}}(T):=\int_{\operatorname{dim}} \phi_{\mathcal{W}}^{\mathrm{eq}}=\sum_{n \geq 0} \frac{\int_{\operatorname{Mat}_{k}(n, n)} \phi_{W_{n}}^{\mathrm{eq}}}{\mathbb{L}^{-n^{2} / 2}\left[\mathrm{Gl}_{k}(n)\right]} T^{n}
$$

in $\mathrm{K}^{\widehat{\mu}}\left(\mathrm{St}^{\text {aff }} / \mathbb{N}\right)$. To compute the series $\Phi_{W}(T)$ and $\Phi_{W}^{\mathrm{eq}}(T)$ we use the stack

$$
\operatorname{Hilb}\left(\mathbb{A}_{k}^{1}\right)=\coprod_{n \geq 0} H_{n} / \mathrm{Gl}_{k}(n) \cong \coprod_{n \geq 0} \mathbb{A}_{k}^{n},
$$


with $H_{n}:=\left\{(A, v) \in \operatorname{Mat}_{k}(n, n) \times \mathbb{A}_{k}^{n} \mid \operatorname{span}_{k}\left(v, A v, \ldots, A^{n-1} v\right)=k^{n}\right\}$ equipped with the obvious $\mathrm{Gl}_{k}(n)$-action. Let $\mathbb{G}_{m}$ act on $H_{n}$ by scalar multiplication and on $\mathbb{A}_{k}^{n}$ with weights $1, \ldots, n$. The isomorphism (8) is induced by the $\mathrm{Gl}_{k}(n)$-principal bundle $q_{n}: H_{n} \ni(A, v) \longmapsto\left(\operatorname{tr} A^{1}, \ldots, \operatorname{tr} A^{n}\right) \in \mathbb{A}_{k}^{n}$. If for any $A \in \operatorname{Mat}_{k}(n, n)$ we express $\operatorname{tr} W(A)$ in terms of $\operatorname{tr}\left(A^{i}\right)$ with $i \in\{1, \ldots, n\}$, we obtain functions $f_{n}: \mathbb{A}_{k}^{n} \rightarrow \mathbb{A}_{k}^{1}$ making the diagram

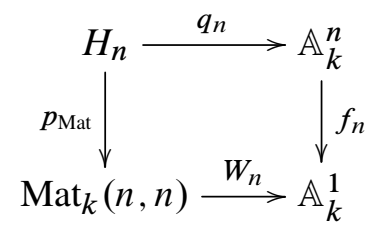

commutative and $\mathbb{G}_{m}$-equivariant if $W$ is homogeneous.

Lemma 4.1 Using the notation just introduced, one has

$$
\Phi_{W}(\mathbb{L} T) / \Phi_{W}(T)=\sum_{n \geq 0} \int_{\mathbb{A}_{k}^{n}} \phi_{f_{n}}\left(\mathbb{L}^{1 / 2} T\right)^{n},
$$

and

$$
\Phi_{W}^{\mathrm{eq}}(\mathbb{L} T) / \Phi_{W}^{\mathrm{eq}}(T)=\sum_{n \geq 0} \int_{\mathbb{A}_{k}^{n}} \phi_{f_{n}}^{\mathrm{eq}}\left(\mathbb{L}^{1 / 2} T\right)^{n} \quad \text { for homogeneous } W
$$

in $\mathrm{K}^{\widehat{\mu}}\left(\mathrm{St}^{\mathrm{aff}} / \mathbb{N}\right) \cong \mathrm{K}^{\widehat{\mu}}(\operatorname{Var} / k)_{\mathrm{Gl}_{k}} \llbracket T \rrbracket$.

Proof The key observation is the following formula in the (equivariant) Hall algebra $\mathrm{K}^{\left(\mathbb{G}_{m}\right)}\left(\mathrm{St}^{\mathrm{aff}} / \mathcal{X}\right)$ first observed by Reineke (see [8, Lemma 5.1]):

$\left[\frac{\operatorname{Mat}_{k}(N, N) \times \mathbb{A}_{k}^{N}}{\mathrm{Gl}_{k}(N)} \stackrel{p_{\text {Mat }}}{\longrightarrow} \mathcal{X}\right]=\sum_{n=0}^{N}\left[\frac{H_{n}}{\mathrm{Gl}_{n}} \stackrel{p_{\text {Mat }}}{\longrightarrow} \mathcal{X}\right] \star\left[\frac{\operatorname{Mat}_{k}(N-n, N-n)}{\mathrm{Gl}_{k}(N-n)} \hookrightarrow \mathcal{X}\right]$

We apply the algebra homomorphisms

$$
\begin{gathered}
\int_{\operatorname{dim}}^{\phi_{\mathcal{W}}}: \mathrm{K}\left(\mathrm{St}^{\mathrm{aff}} / \mathcal{X}\right) \ni[\mathcal{Z} \stackrel{\pi}{\rightarrow} \mathcal{X}] \longmapsto \int_{\operatorname{dim} \circ \pi} \pi^{*} \phi_{\mathcal{W}} \in \mathrm{K}^{\widehat{\mu}}\left(\mathrm{St}^{\mathrm{aff}} / \mathbb{N}\right), \\
\int_{\operatorname{dim}}^{\phi_{\mathcal{W}}^{\mathrm{eq}}}: \mathrm{K}^{\mathbb{G}_{m}}\left(\mathrm{St}^{\mathrm{aff}} / \mathcal{X}\right) \ni[\mathcal{Z} \stackrel{\pi}{\rightarrow} \mathcal{X}] \longmapsto \int_{\mathcal{Z}} \phi_{\mathcal{W} \circ \pi}^{\mathrm{eq}} \in \mathrm{K}^{\widehat{\mu}}\left(\mathrm{St}^{\mathrm{aff}} / \mathbb{N}\right)
\end{gathered}
$$

from the (equivariant) Hall algebras to $\mathrm{K}^{\widehat{\mu}}\left(\mathrm{St}^{\mathrm{aff}} / \mathbb{N}\right)$. These are as constructed in [15], but working over quivers the Hall algebra is much simplified, and so is the proof that the map is an algebra homomorphism, resting entirely on the proof that the 
"integral identity" of [15, Section 4.4] holds. For a proof of this, see [19] or [20]. By Proposition 2.10(3),

$$
\int_{\operatorname{dim}}^{\phi_{\mathcal{W}}}\left[\frac{\operatorname{Mat}_{k}(N, N) \times \mathbb{A}_{k}^{N}}{\mathrm{Gl}_{k}(N)} \stackrel{p_{\text {Mat }}}{\longrightarrow} \mathcal{X}\right]=\mathbb{L}^{N} \int_{\operatorname{dim}}^{\phi_{\mathcal{W}}}\left[\frac{\operatorname{Mat}_{k}(N, N)}{\mathrm{Gl}_{k}(N)} \hookrightarrow \mathcal{X}\right]
$$

and

$$
\int_{\operatorname{dim}}^{\phi_{\mathcal{W}}}\left[H_{n} / \mathrm{Gl}_{k}(n) \stackrel{p_{\mathrm{Mat}}}{\longrightarrow} \mathcal{X}\right]=\mathbb{L}^{n / 2} \int_{\mathbb{A}_{k}^{n}} \phi_{f_{n}},
$$

and similarly for the equivariant version. Multiplying with $T^{N}$ and summing over $N \geq 0$ proves the lemma. Notice that the lemma can also be seen as a special wallcrossing formula (see [23]).

To compute the integral $\mathbb{L}^{n / 2} \int_{\mathbb{A}_{k}^{n}} \phi_{f_{n}}$ we restrict ourselves firstly to the case $W=t^{d}$ of normalized homogeneous potentials. By Theorem 3.1, $\Phi_{W}(T)=\Phi_{W}^{\mathrm{eq}}(T)$, and

$$
\mathbb{L}^{n / 2} \int_{\mathbb{A}_{k}^{n}} \phi_{f_{n}}=\mathbb{L}^{n / 2} \int_{\mathbb{A}_{k}^{n}} \phi_{f_{n}}^{\mathrm{eq}}
$$

follows from the previous lemma. Notice that $\operatorname{Sym}^{n} \mathbb{A}_{k}^{1} \cong \mathbb{A}_{k}^{n}$, induced by the map

$$
\tilde{q}_{n}: \mathbb{A}_{k}^{n} \ni\left(z_{1}, \ldots, z_{n}\right) \longmapsto\left(z_{1}+\cdots+z_{n}, \ldots, z_{1}^{n}+\cdots+z_{n}^{n}\right) \in \mathbb{A}_{k}^{n} .
$$

An easy calculation shows that $f_{n} \circ \widetilde{q}_{n}\left(z_{1}, \ldots, z_{n}\right)=z_{1}^{d}+\cdots+z_{n}^{d}$; in other words, $f_{n}=\operatorname{Sym}_{+}^{n}(W)$. We finally obtain

$$
\mathbb{L}^{n / 2} \int_{\mathbb{A}_{k}^{n}} \phi_{f_{n}}=\mathbb{L}^{n / 2} \int_{\mathbb{A}_{k}^{n}} \phi_{f_{n}}^{\mathrm{eq}}=\sigma^{n}\left(\mathbb{L}^{1 / 2} \int_{\mathbb{A}_{k}^{1}} \phi_{W}^{\mathrm{eq}}\right)=\sigma^{n}\left(1-\left[\mu_{d}\right]\right),
$$

with $\mu_{d}$ carrying the obvious $\widehat{\mu}$-action.

Theorem 4.2 The motivic Donaldson-Thomas invariants for the one-loop quiver with homogeneous potential $W=t^{d}$ are given by

$$
\Omega_{n}= \begin{cases}\mathbb{L}^{-1 / 2}\left(1-\left[\mu_{d}\right]\right) & \text { for } n=1 \\ 0 & \text { otherwise. }\end{cases}
$$

Proof Using the previous lemma and our calculations, we get

$$
\begin{aligned}
\operatorname{Sym}\left(\sum_{n \geq 1} \frac{\mathbb{L}^{n}-1}{\mathbb{L}^{1 / 2}-\mathbb{L}^{-1 / 2}} \Omega_{n} T^{n}\right)=\Phi_{W}(\mathbb{L} T) / \Phi_{W}(T) & =\sum_{n \geq 0} \sigma^{n}\left(1-\left[\mu_{d}\right]\right) T^{n} \\
& =\operatorname{Sym}\left(\left(1-\left[\mu_{d}\right]\right) T\right),
\end{aligned}
$$

and the theorem follows by comparing coefficients. 
Let us now come back to the case of general potentials $0 \neq W \in k[t]$ and let $W^{\prime}=$ $c \prod_{i=1}^{r}\left(t-a_{i}\right)^{d_{i}-1}$ be the prime decomposition of $W^{\prime}$ into linear factors, with $c \in k^{\times}$, $1<d_{i} \in \mathbb{N}$ and $a_{i} \in k$ for all $1 \leq i \leq r$. Hence, the Grothendieck group of the abelian category of sheaves supported on the zero scheme of $W^{\prime}$ is $\mathbb{Z}^{r}$ with effective cone $\mathbb{N}^{r}$ spanned by the classes $\vec{e}_{i}$ of skyscraper sheaves of length one supported at $a_{i} \in \mathbb{A}_{k}^{1}$ $(i \in\{1, \ldots, r\})$, or equivalently by one-dimensional representations with eigenvalue $a_{i}$. The monoid homomorphism $\operatorname{dim}: \operatorname{Crit}(\mathcal{W}) \longrightarrow \mathbb{N}$ factorizes as

$$
\operatorname{dim}: \operatorname{Crit}(\mathcal{W}) \stackrel{\mathrm{cl}}{\longrightarrow} \mathbb{N}^{r} \stackrel{+}{\longrightarrow} \mathbb{N},
$$

with $\operatorname{cl}(V)$ being the class of the representation of $V$ in the Grothendieck group. This allows us to define refined Donaldson-Thomas invariants by means of

$\int_{\mathrm{cl}} \phi_{\mathcal{W}}=\operatorname{Sym}\left(\frac{1}{\mathbb{L}^{1 / 2}-\mathbb{L}^{-1 / 2}} \sum_{\vec{n}=\left(n_{1}, \ldots, n_{r}\right) \in \mathbb{N}^{r} \backslash\{0\}} \Omega_{\vec{n}} T_{1}^{n_{1}} \cdots T_{r}^{n_{r}}\right)=: \Phi_{W}\left(T_{1}, \ldots, T_{r}\right)$

in $\mathrm{K}^{\widehat{\mu}}\left(\mathrm{St}^{\text {aff }} / \mathbb{N}^{r}\right)$, where we denote the element $\left[\operatorname{Spec}(k) \rightarrow \vec{e}_{i}\right]$ by $T_{i}$. For any $1 \leq i \leq r$ let us write $\mathfrak{M}^{(i)}$ for the substack cl${ }^{-1}\left(\mathbb{N} \vec{e}_{i}\right)$ parametrizing sheaves on $\left\{a_{i}\right\} \subset \mathbb{A}_{k}^{1}$. Obviously

$$
\mathfrak{M} \cong \prod_{i=1}^{r} \mathfrak{M}^{(i)}
$$

by taking direct sums. However, to compute the motivic vanishing cycle $\phi_{\mathcal{W}}$ we use different embeddings on each side. Indeed, the vanishing cycle on the left-hand side restricted to $\mathrm{cl}^{-1}\left(n_{1}, \ldots, n_{r}\right)=: \mathfrak{M}_{\vec{n}}$ is computed by means of the embedding $\mathfrak{M}_{\vec{n}} \subset \operatorname{Mat}_{k}(N, N) / \mathrm{Gl}_{k}(N)$ with $N=n_{1}+\cdots+n_{r}$, whereas on the right-hand side we use the embedding

$$
\prod_{i=1}^{r} \mathfrak{M}_{n_{i} \vec{e}_{i}} \subset \prod_{i=1}^{r} \operatorname{Mat}_{k}\left(n_{i}, n_{i}\right) / \mathrm{Gl}_{k}\left(n_{i}\right) .
$$

However, one can prove the product formula

$$
\int_{\mathrm{cl}} \phi_{\mathcal{W}}=\left.\prod_{i=1}^{r} \int_{\mathrm{cl}} \phi_{\mathcal{W}}\right|_{\mathfrak{M}^{(i)}}
$$

Indeed, as there are neither extensions nor morphisms between representations $V \in \mathfrak{M}^{(i)}$ and $V^{\prime} \in \mathfrak{M}^{(j)}$ for $i \neq j$, we have

$$
[\mathfrak{M} \rightarrow \mathfrak{M}]=\left[\mathfrak{M}^{(1)} \rightarrow \mathfrak{M}\right] \star \cdots \star\left[\mathfrak{M}^{(r)} \rightarrow \mathfrak{M}\right]
$$


in the Hall algebra $\mathrm{K}\left(\mathrm{St}^{\text {aff }} / \mathfrak{M}\right)$. To prove formula (9) we again apply the refined algebra homomorphism $\int_{\mathrm{cl}}^{\phi_{\mathcal{W}}}$ from the Hall algebra to $\mathrm{K}^{\widehat{\mu}}\left(\mathrm{St}^{\text {aff }} / \mathbb{N}^{r}\right)$.

For the computation of the right-hand side in the product formula (9) we make use of the following lemma.

Lemma 4.3 For any $i \in\{1, \ldots, r\}$, let $d_{i}$ be defined as above. Then

$$
\left.\int_{\mathrm{cl}} \phi_{W}\right|_{\mathfrak{M}^{(i)}}=\Phi_{t^{d_{i}}}\left(T_{i}\right)=\operatorname{Sym}\left(\frac{1-\left[\mu_{d_{i}}\right]}{\mathbb{L}-1} T_{i}\right) \text {. }
$$

Proof Fix $i \in\{1, \ldots, r\}$ and $0<n \in \mathbb{N}$. By translation we can assume $a_{i}=0$ and $W=\sum_{p \geq 0} b_{p} t^{p+d_{i}}$ with $b_{0} \neq 0$ but $b_{p}=0$ for $p \gg 0$. Let $\tilde{W}(t)=t^{d_{i}}$. By solving the recursive equations

$$
\sum_{\substack{m_{0}+m_{1}+\cdots=d_{i} \\
m_{1}+2 m_{2}+\cdots=p}}\left(\begin{array}{c}
d_{i} \\
m_{0}, m_{1}, \ldots
\end{array}\right) a_{0}^{m_{0}} a_{1}^{m_{1}} \cdots=b_{p}
$$

starting with a $d_{i}^{\text {th }}$ root $a_{0}$ of $b_{0}$, one finds a power series $\theta(t)=t\left(a_{0}+a_{1} t+\cdots\right)$ with $\theta(t)^{d_{i}}=\tilde{W}(\theta(t))=W(t)$.

The component $\mathfrak{M}_{n \vec{e}_{i}}$ is given by the quotient stack $C / \mathrm{Gl}(n)$, with

$$
C=\left\{A \in \operatorname{Mat}_{k}(n, n) \mid A^{d_{i}-1}=0\right\}
$$

as

$$
d W_{n}(A): \operatorname{Mat}_{k}(n, n) \ni H \longmapsto \operatorname{tr}\left(H A^{d_{i}-1} G(A)\right) \in \mathbb{A}_{k}^{1}
$$

with $G \in k[t]$ having nonzero constant term. As the map $A \mapsto A^{m\left(d_{i}-1\right)}$ is in the $m^{\text {th }}$ power of the defining ideal of $C$, we obtain a well-defined isomorphism $\theta_{n}: \widehat{C} \rightarrow \widehat{C}$ on the formal neighborhood of $C$, ie the formal completion of $\operatorname{Mat}_{k}(n, n)$ along $C$. Moreover, the restriction of the function $\tilde{W}_{n}: A \mapsto \operatorname{tr} A^{d_{i}}$ to $\widehat{C}$ composed with $\theta_{n}$ coincides with the restriction of $W_{n}$ to $\widehat{C}$. As any arc of length $l$ in $\operatorname{Mat}_{k}(n, n)$ with constant term in $C$ is actually an arc of length $l$ in $\widehat{C}, \theta_{n}$ induces isomorphisms

$$
\mathcal{L}_{l}\left(\theta_{n}\right):\left.\left.\mathcal{L}_{l}\left(\operatorname{Mat}_{k}(n, n)\right)\right|_{C} \stackrel{\sim}{\longrightarrow} \mathcal{L}_{l}\left(\operatorname{Mat}_{k}(n, n)\right)\right|_{C},
$$

with $\mathcal{L}_{l}\left(\widetilde{W}_{n}\right) \circ \mathcal{L}_{l}\left(\theta_{n}\right)=\mathcal{L}_{l}\left(W_{n}\right)$ for any $l>0$. Hence

$$
\int_{C} Z_{W_{n}}^{\mathrm{eq}}\left(T_{i}\right)=\int_{C} Z_{\widetilde{W}_{n}}^{\mathrm{eq}}\left(T_{i}\right)
$$

with the notation from Section 2, and $\int_{C} \phi_{W_{n}}=\int_{C} \phi_{\widetilde{W}_{n}}$ follows, proving the first equality. The second equality is a direct consequence of Theorem 4.2. 
Combining the lemma with the preceding arguments proves our main theorem.

Theorem 4.4 For $W \in k[T]$, let $W^{\prime}=c \prod_{i_{1}}^{r}\left(t-a_{i}\right)^{d_{i}-1}$, with $c \in k^{\times}, 1<d_{i} \in \mathbb{N}$ and $a_{i} \in k$ for all $1 \leq i \leq r$, as before. Define the Donaldson-Thomas invariants $\Omega_{\vec{n}} \in \mathrm{K}^{\widehat{\mu}}\left(\mathrm{St}^{\text {aff }} / k\right)$ for any $r$-tuple $\left(n_{1}, \ldots, n_{r}\right) \in \mathbb{N}^{r}$ as above. Then

$$
\Omega_{\vec{n}}= \begin{cases}\mathbb{L}^{-1 / 2}\left(1-\left[\mu_{d_{i}}\right]\right) & \text { for } \vec{n}=\vec{e}_{i}(1 \leq i \leq r), \\ 0 & \text { otherwise. }\end{cases}
$$

In particular, $\Omega_{\vec{n}}$ is in the image of $\mathrm{K}^{\widehat{\mu}}(\operatorname{Var} / k)\left[\mathbb{L}^{-1 / 2}\right]$ in $\mathrm{K}^{\widehat{\mu}}\left(\mathrm{St}^{\mathrm{aff}} / k\right)$.

\section{References}

[1] K Behrend, Donaldson-Thomas type invariants via microlocal geometry, Ann. of Math. 170 (2009) 1307-1338 MR2600874

[2] K Behrend, J Bryan, B Szendrői, Motivic degree zero Donaldson-Thomas invariants, Invent. Math. 192 (2013) 111-160 MR3032328

[3] A Białynicki-Birula, Some theorems on actions of algebraic groups, Ann. of Math. 98 (1973) 480-497 MR0366940

[4] A Białynicki-Birula, Some properties of the decompositions of algebraic varieties determined by actions of a torus, Bull. Acad. Polon. Sci. Sér. Sci. Math. Astronom. Phys. 24 (1976) 667-674 MR0453766

[5] J Denef, F Loeser, Geometry on arc spaces of algebraic varieties, from: "European Congress of Mathematics, Volume I", (C Casacuberta, R M Miró-Roig, J Verdera, S Xambó-Descamps, editors), Progr. Math. 201, Birkhäuser, Basel (2001) 327-348 MR1905328

[6] J Denef, F Loeser, Lefschetz numbers of iterates of the monodromy and truncated arcs, Topology 41 (2002) 1031-1040 MR1923998

[7] T Ekedahl, The Grothendieck group of algebraic stacks arXiv:0903.3143

[8] J Engel, M Reineke, Smooth models of quiver moduli, Math. Z. 262 (2009) 817-848 MR2511752

[9] D Joyce, Configurations in abelian categories, I: Basic properties and moduli stacks, Adv. Math. 203 (2006) 194-255 MR2231046

[10] D Joyce, Configurations in abelian categories, II: Ringel-Hall algebras, Adv. Math. 210 (2007) 635-706 MR2303235

[11] D Joyce, Configurations in abelian categories, III: Stability conditions and identities, Adv. Math. 215 (2007) 153-219 MR2354988

[12] D Joyce, Motivic invariants of Artin stacks and "stack functions", Q. J. Math. 58 (2007) 345-392 MR2354923 
[13] D Joyce, Configurations in abelian categories, IV: Invariants and changing stability conditions, Adv. Math. 217 (2008) 125-204 MR2357325

[14] D Joyce, Y Song, A theory of generalized Donaldson-Thomas invariants, Mem. Amer. Math. Soc. 1020, Amer. Math. Soc. (2012) MR2951762

[15] M Kontsevich, Y Soibelman, Stability structures, motivic Donaldson-Thomas invariants and cluster transformations arXiv:0811.2435

[16] M Kontsevich, Y Soibelman, Motivic Donaldson-Thomas invariants: Summary of results, from: "Mirror symmetry and tropical geometry", (R Castaño-Bernard, Y Soibelman, I Zharkov, editors), Contemp. Math. 527, Amer. Math. Soc. (2010) 55-89 MR2681792

[17] M Kontsevich, Y Soibelman, Cohomological Hall algebra, exponential Hodge structures and motivic Donaldson-Thomas invariants, Commun. Number Theory Phys. 5 (2011) 231-352 MR2851153

[18] A Kresch, Cycle groups for Artin stacks, Invent. Math. 138 (1999) 495-536 MR1719823

[19] Q T Lê, Proofs of the integral identity conjecture over algebraically closed fields, Duke Math. J. 164 (2015) 157-194 MR3299104

[20] D Maulik, Motivic residues and Donaldson-Thomas theory, preprint, available from the author

[21] A Morrison, S Mozgovoy, K Nagao, B Szendrői, Motivic Donaldson-Thomas invariants of the conifold and the refined topological vertex, Adv. Math. 230 (2012) 2065-2093 MR2927365

[22] S Mozgovoy, On the motivic Donaldson-Thomas invariants of quivers with potentials, Math. Res. Lett. 20 (2013) 107-118 MR3126726

[23] S Mozgovoy, Wall-crossing formulas for framed objects, Q. J. Math. 64 (2013) 489-513 MR3063519

[24] R P Thomas, A holomorphic Casson invariant for Calabi-Yau 3-folds, and bundles on K3 fibrations, J. Differential Geom. 54 (2000) 367-438 MR1818182

Section de mathématiques, École Polytechnique Fédérale de Lausanne Station 8, Bâtiment MA, CH-1015 Lausanne, Switzerland

Fachbereich C-Mathematik und Naturwissenschaften, Bergische Universität Wuppertal Gaussstrasse 20, D-42119 Wuppertal, Germany

nicholas.davison@epfl.ch, sven@math.uni-bonn.de

Proposed: Richard Thomas

Received: 30 January 2013

Seconded: Jim Bryan, Lothar Göttsche

Revised: 26 August 2014 
